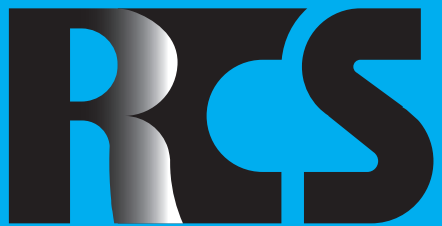

Depósito legal ppi $201502 Z U 4662$

Esta publicación científica en formato

digital es continuidad de la revista impresa

Depósito Legal: pp $197402 Z U 789$

ISSN: 1315-9518

\title{
Revista de Ciencias Sociales
}

Universidad del Zulia. Revista de la Facultad de Ciencias Económicas y Sociales Vol. XXV. Número especial 1, 2019

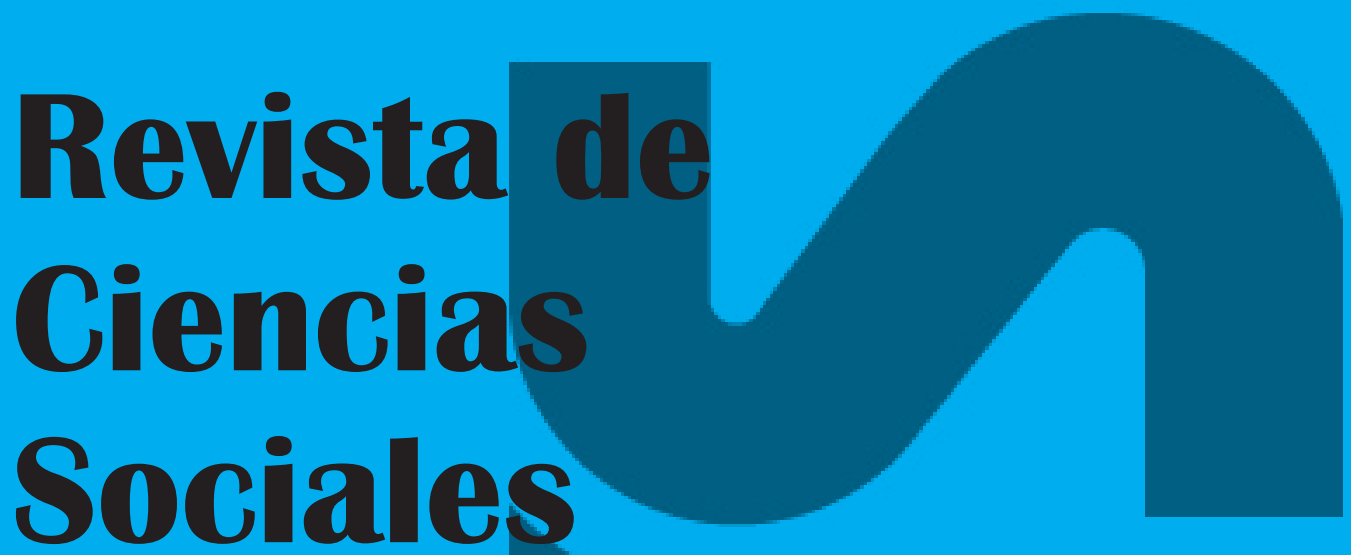




\title{
Desarrollo económico local en Ecuador: Relación entre producto interno bruto y sectores económicos
}

\author{
Proaño Chaca, Sonnia Alexandra* \\ Quiñonez Alvarado, Erika Sonia** \\ Molina Villacís, Carolina Jennifer*** \\ Mejía Flores, Omar Gabriel ${ }^{* * * *}$
}

\section{Resumen}

El desarrollo económico local es un proceso sistemático que involucra diversos sectores, tales como: Público, privado, asociaciones, entre otros; cuya finalidad radica en el aumento, a la par, de la calidad y el nivel de vida de los habitantes. En Ecuador, este proceso tiene un extenso camino por recorrer, debido a la presencia de indicadores socioeconómicos en cifras negativas en diversos cantones del territorio nacional. En efecto, esta investigación tiene como objetivo describir las principales variables sociales y económicas de los cantones Daule, Nobol y Santa Lucía, las cuales se circunscriben en el proceso del desarrollo económico local. La metodología aplicada fue bajo un diseño no experimental de tipo descriptivo y exploratorio. Se elaboró un modelo de datos de panel con la finalidad de evaluar la sensibilidad de los sectores económicos, considerando los efectos no observables, en el crecimiento del Producto Interno Bruto de dichos cantones. Como resultado, los indicadores socioeconómicos reflejan el escaso desarrollo económico que posee aquel distrito, concluyendo que es necesario el diseño e implementación de políticas públicas efectivas y partici-

Magister en Planificación de Proyectos en Desarrollo rural (Universidad Politécnica de Madrid - España). Magister en Tributación e Ingeniería Comercial (Universidad Politécnica del Litoral, Guayaquil - Ecuador). Profesora titular auxiliar 1 de la Universidad Politécnica Salesiana, sede Guayaquil, Ecuador. Email: sonia.proaño@ups.edu.ec ORCID: https://orcid.org/0000-0001-5299-106X

** Doctora(c) en Contabilidad (Universidad de Tumbes). Magister en Finanzas y Proyectos Corporativos. Contadora Pública de la Universidad de Guayaquil. Profesora titular tiempo completo de la Universidad de Guayaquil, Ecuador. Email: erika. quiñoneza@ug.edu.ec ORCID: https://orcid.org/0000-0002-3520-5183

*** Magister en Economía y Dirección de Empresas (ESPOL). Ingeniera en Gestión Empresarial Internacional (ESPOL). Profesora de la Universidad de Guayaquil, Ecuador. Email: carolina.molinav@ug.edu.ec ORCID: https://orcid.org/0000-0002$7241-8882$

**** Doctor(c) en Administración Estratégica de Empresas (Pontificia Universidad Católica del Perú). Doctor(c) en Ciencias Económicas (Universidad del Zulia, Venezuela). Magíster en Tributación y Finanzas. Ingeniero Comercial (Universidad de Guayaquil). Profesor Ocasional Universidad de Guayaquil, Ecuador. Email: omar.mejiaf@ug.edu.ec ORCID: https://orcid. org/0000-0001-5129-8933 
pativas para revertir dicha situación; además, el modelo arroja una mayor sensibilidad del Producto Interno Bruto hacia el comercio al por mayor y menor.

Palabras clave: Desarrollo económico local; modelo de datos de panel; sensibilidad; sectores económicos; indicadores socioeconómicos.

\title{
Local economic development in Ecuador: Relationship between gross domestic product and economic sectors
}

\begin{abstract}
Local economic development is a systematic process that involves various sectors, such as: Public, private, associations, among others; whose purpose lies in increasing, at the same time, the quality and standard of living of the inhabitants. In Ecuador, this process has a long way to go, due to the presence of socioeconomic indicators in negative figures in various cantons of the national territory. Indeed, this research aims to describe the main social and economic variables of the cantons Daule, Nobol and Santa Lucía, which are circumscribed in the process of local economic development. The methodology applied was under a non-experimental design of descriptive and exploratory type. A panel data model was developed in order to assess the sensitivity of the economic sectors, considering the unobservable effects, on the growth of the Gross Domestic Product of these cantons. As a result, socioeconomic indicators reflect the poor economic development that the district possesses, concluding that it is necessary to design and implement effective and participatory public policies to reverse this situation; In addition, the model shows a greater sensitivity of the Gross Domestic Product towards wholesale and retail trade.
\end{abstract}

Keywords: Local economic development; panel data model; sensitivity; economic sectors; socioeconomic indicators.

\section{Introducción}

A mediados del siglo XVIII cuando se daba inicio al camino hacia la Revolución Industrial, bajo una ideología netamente liberalista, el enfoque que tenía el desarrollo económico era, netamente, basado en la empresarialidad, lo cual demandaba una rápida urbanización, en efecto, una migración del campo hacia las grandes ciudades. En referencia a aquello, Alburquerque (2004) hace mención a la importancia que han tenido los niveles de industrialización en las naciones, para medir el desarrollo en sus habitantes y no se ha tomado en cuenta la territorialización del desarrollo. En otras palabras, considera que el enfoque territorial del desarrollo económico ha sufrido una marginación teórica durante mucho tiempo, es decir que, desde una perspectiva historicista, los niveles de desarrollo han estado explicado solo por los niveles de industrialización.

En conjunción con el proceso de la industrialización, a nivel mundial ha tomado mayor fuerza el paradigma de la globalización, lo cual obliga a los mercados a ser cada vez más competitivos y eficiente, puesto que una de las características teóricas que tiene este paradigma es la apertura y unificación de todos los mercados a nivel mundial, por lo que las generaciones de políticas económicas eficientes a nivel de nación será indispensable para competir en mercados internacionales, algunas de las políticas que más se ha usado, desde 
antaño hasta la actualidad, referentes al sector externo, han sido: Medidas proteccionistas (aranceles elevados), licencias de importación y subvenciones a exportaciones.

Paradójicamente, Chang (2004; 2010) menciona que los países que suponían tener un sistema de libre mercado y comercio, en niveles de desarrollo similares a los países, actualmente atrasados, imponían medidas proteccionistas con la finalidad de proteger a la industria/sector incipiente en sus economías y llegasen a desarrollarse de tal manera que pudieran competir en un mercado cada vez más globalizado, y para ese entonces, ya se inclinaban por un libre comercio y un mercado no regulado.

En efecto, Aghón, Alburquerque $\mathrm{y}$ Cortés (2001) consideran que el enfoque de desarrollo económico local se postula para responder a los desafíos que imponen los mercados internacionales respecto a los niveles elevados de competitividad, y una de las formas para atender dichos criterios es asegurar la inserción de innovaciones en diversas aristas, tales como: Tecnologías, de gestión, sociales e institucionales dentro de los procesos productivos locales.

Al respecto, la economía ecuatoriana sigue dependiendo de los ingresos percibidos por los productos del sector primario, es decir no conllevan un valor agregado considerable. En otras palabras, la estructura productiva no ha tenido un cambio que permita darle una mejora en los niveles de competitividad para así responder de forma positiva a las exigencias que dictan los mercados internacionales.

Además, la dependencia económica del Ecuador hacia los commodities hace que la economía nacional no sea sostenible a largo plazo, debido a que los precios de dichos bienes están juzgados y determinados por el mercado internacional cada vez más amplio y con bienes sustitutos con mayor competitividad, lo cual hace que caigan los precios de los bienes producidos en la nación; sin embargo, una de las alternativas a estos shocks externos no controlables, es el desarrollo bajo un enfoque endógeno, fortaleciendo las economías locales con mejores instituciones, mayor dotación tecnológica e innovaciones en la gestión social. Es por eso, que la implementación de nuevas políticas que estén basadas bajo el enfoque del desarrollo económico local, es indispensable para que la economía de la nación empiece a tomar el rumbo hacia la sostenibilidad.

En ese sentido, los cantones por estudiar en esta investigación son el reflejo de cómo está estructurada la economía nacional. En adición, es importante resaltar que estas ciudades poseen características similares en lo que a actividades económicas principales se refiere, aunque guardan distancia en los niveles de ingresos que perciben. A continuación, se plasma una breve reseña de las principales características que poseen dichas zonas geográficas:

El Cantón Daule, perteneciente a la Provincia del Guayas, tiene una de las cantonizaciones más antiguas, la cual data de 1820; por otro lado, mantiene la mayor producción agrícola de la Región Litoral dentro de la Cuenca Hidrográfica del Río Guayas. Además, el Plan de Desarrollo y Ordenamiento Territorial (PDYOT) de Daule, 2015 (Gobierno Autónomo Descentralizado [GAD] de la Ilustre Municipalidad del Cantón Daule, 2015), considera que el cantón cuenta con una población relativamente equilibrada en sus dos áreas: Urbana y rural.

El Cantón Nobol, perteneciente a la Provincia del Guayas, tuvo su cantonización en el año 1992, mantiene límites con el Cantón Daule, Guayaquil, Río Daule e Isidro Ayora. Según PDYOT para el año 2015, el cantón contaba con aproximadamente 23.218 habitantes (GAD Municipal del Cantón de Nobol, 2015). Y, por último, el Cantón Santa Lucía, en el PDYOT de 2014-2025, se menciona que el cantón cuenta con recursos naturales muy similares a los dos cantones ya descritos. Su principal actividad se remonta a la producción agrícola, específicamente, el cultivo del arroz (GAD Municipal del Cantón de Santa Lucía, 2016).

Por todo lo antes expuesto, el objetivo principal de esta investigación es explorar el nivel de desarrollo económico de los mencionados cantones, mediante un análisis 
descriptivo de las principales variables sociales y económicas, es decir desde una perspectiva integral.

La distribución de la investigación se detalla a continuación: En primer lugar, se expone una revisión literaria en la que se sustenta el trabajo; a posteriori, se muestra la metodología, en la cual se detallan los datos a utilizar y el modelo a estimar; luego, se presentan los resultados encontrados en las fuentes oficiales, mediante un análisis descriptivo-situacional de las principales variables socioeconómicas, y además, se presenta la estimación del modelo, seleccionado previamente, mediante los contraste más robustos.

\section{Revisión literaria: Marco teórico del desarrollo económico local}

El Desarrollo Económico Local (DEL), es un enfoque que ha surgido acorde a los problemas coyunturales económicos y sociales que se han presentado recientemente en los diferentes países; pues, estas dificultades se han incrementado de acuerdo a diversos factores, tales como: Cambios en el mercado laboral, el incremento de la economía informal, la inexistencia de ventajas competitivas, y la falta de políticas descentralizadas por parte de los gobiernos de turno; estas características han hecho como consecuencia la necesidad de fomentar un nuevo tipo de desarrollo económico enfocado en el crecimiento endógeno (Quispe, Ayaviri y Maldonado, 2018), en la generación de competitividad, un cambio estructural, en lo que respecta al ámbito territorial y en el bienestar de una sociedad local.

En este sentido y tal como lo mencionan Quispe, et al. (2018), “elementos como la adaptación al cambio tecnológico, sus instituciones, la productividad, son considerados en primera línea para alcanzar escenarios que contribuyen en el progreso y el bienestar de la población" (p.66).

En la mayor parte de países latinoamericanos, los últimos 10 años han sido presididos por una corriente de izquierda o direccionada hacia un sistema socialista, por lo que la centralización, es una de las características principales de aquella corriente; países como Bolivia, Venezuela, Brasil y Ecuador, han adoptado políticas económicas con enfoques de goteo hacia abajo, por lo que no se han desarrollado óptimamente las economías a nivel local, cabe destacar que existen algunas excepciones, pero esto se da porque dichas políticas son coherentes con algunas regiones.

En este sentido, las políticas a nivel central son importantes en el enfoque DEL, puesto que forma parte del marco institucional al cual debe ajustarse cualquier medida, que quisiera implementarse en niveles más bajos de gobernabilidad. Por otro lado, la consciencia de las sociedades locales, es herramienta primordial para la generación de actividades innovadoras, que permitan maximizar los ingresos que perciben, por lo que "las actitudes por desarrollarse y/o progresar marcan la base del enfoque del desarrollo económico local" (Maldonado, 2000, p.10), concentrando esto al objetivo de estimular la economía local con la explotación de recursos que sean locales y potenciales para así incorporarlos a las diferentes oportunidades en el resto del mundo.

En contraste, Vázquez (2000) realiza un análisis más integral y destaca que la base del desarrollo económico local radica en la optimización de las ventajas comparativas, que poseen los diferentes territorios que conforman la región y/o nación, a su vez se enfoca el desarrollo económico local bajo diversas dimensiones, tal como se muestra en la Figura I. 


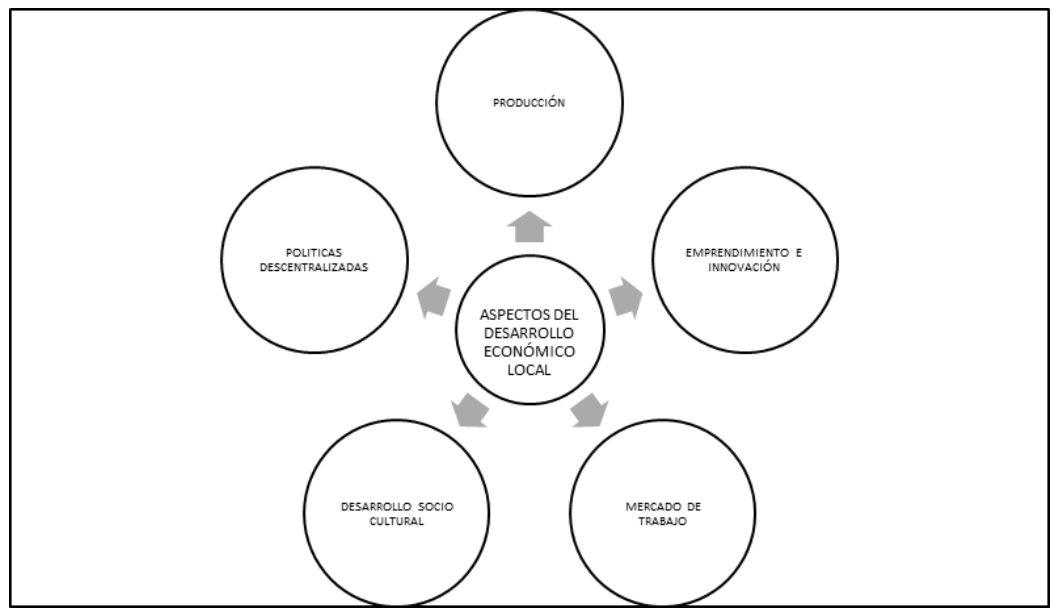

Fuente: Elaboración propia, 2019. Adaptado de Vázquez (2000).

Figura I. Aspectos del desarrollo económico local

Respecto a la optimización de las ventajas comparativas que poseen los diversos territorios en una nación, dichas ventajas deben ir concatenadas con una dotación tecnológica idónea y la presencia de innovaciones en todos los procesos productivos; en otras palabras, para alcanzar un desarrollo económico local de un determinado sitio geográfico, se considera la capacidad empresarial de adaptarse a los constantes cambios de innovación, esto se ha visto reflejado en antaño dentro de las sociedades que buscan mejorar su calidad de vida con la captación de nuevas tecnologías, que permitan generar mejores valores agregados a sus productos y/o servicios (Vázquez, 2000); es decir, buscar transformar dichas ventajas naturales o comparativas en verdaderas ventajas competitivas, que consientan una inserción eficiente y consolidación eficaz en los mercados nacionales y extranjeros.

De igual manera, el nivel de emprendimiento guarda una estrecha relación con el comportamiento que tienen los ingresos en la sociedad, es decir que a medida que aumente la tasa de emprendimiento dentro de ésta, es muy probable que la renta per cápita aumente (Durán, 2015). Sin embargo, los emprendimientos están ligados al marco institucional en el que se vayan a desenvolver, y esto es un factor que influye en la consolidación de los mismos. No obstante, la función empresarial no es la única que interviene en un desarrollo económico local sostenible, si bien es cierto lo potencia, pero hay que considerar otras variables que están localizadas desde una perspectiva social, específicamente fomentando y potenciando el capital humano.

En adición, la Organización Internacional del Trabajo (OIT, 2018) y Vázquez (2000), coinciden en que las estrategias de desarrollo económico local son multidisciplinarias, y no sólo de la función empresarial como lo sostiene Durán (2015). Las estrategias para el DEL deben ir enfocadas hacia la protección social, fortalecimiento de la empleabilidad, desarrollo del capital humano, entre otros. Dentro del mercado laboral existen diversas estrategias para promover un trabajo decente, mediante el fortalecimiento institucional local, brindando asistencia técnica para el buen desempeño 
de las dinámicas del mercado de trabajo y su relación directa al entorno empresarial, tomando en consideración también a la innovación; promoviendo además localmente el cooperativismo, la asociatividad y la financiación para nuevos emprendimientos, para que de esta manera se brinde una base concreta al desarrollo económico local.

En sentido estricto, dichas conceptualizaciones están basadas desde una perspectiva integral y/o heterodoxa, en donde prepondera el nivel de ingresos explicados por el aprovechamiento de las ventajas comparativas, que le brinda la ubicación geográfica, el impacto que tiene la dimensión social y cultural en el desarrollo de una sociedad. Cabe destacar que los ingresos que perciben los individuos en la sociedad son importantes, pero "la principal razón de un desarrollo económico local sostenible radica en la actitud que tengan los individuos de la sociedad por progresar en lo económico y mejorar su estatus social (calidad de vida)", tal como lo sugiere (Maldonado, 2000, p.14).

De forma similar, Cárdenas (2002) concluye que el desarrollo económico local es un proceso que se origina de forma diferenciada entre naciones, debido a las articulaciones entre la dimensión territorial, la historia, las estructuras productivas y, sobre todo, la acción diferenciada de los actores sociales y empresariales. De allí, que tal como lo sostiene Delgado (2018), el territorio entendido como el entramado de intereses de todo tipo de una comunidad territorial, puede ser percibido como un agente de desarrollo, siempre que sea posible mantener y desarrollar la integridad y los intereses territoriales en los procesos de crecimiento y cambio estructural en cada país.

En suma, el desarrollo económico local es un proceso que conjuga lo económico, lo histórico y lo social; por esta razón, la creación e implementación de políticas públicas deben tener como eje transversal y participación directa dichos sectores. Los indicadores sociales y económicos, sirven de guía para saber qué políticas son más funcionales y cuáles no.

\section{Metodología}

En el presente estudio de investigación se realiza un análisis descriptivo y exploratorio, desde un diseño no experimental, de los cantones pertenecientes al Distrito 9 del Guayas con la finalidad de conocer sus características, basadas en datos oficiales, sociales y económicas. Además, se evalúa la sensibilidad, mediante un modelo de datos de panel, que tiene el Producto Interno Bruto de los cantones hacia los principales sectores productivos que mantiene su economía; ambos análisis serán herramientas eficientes para medir su nivel de desarrollo económico local desde una perspectiva integral.

Las cifras correspondientes a las variables sociales permiten conocer el nivel de la calidad de vida de los habitantes de los cantones objeto de estudio para el periodo 2007 - 2016. Por otro lado, las variables económicas marcan el nivel de vida de estas ciudades. A continuación, se muestran las variables que son objeto de análisis en esta investigación.

\subsection{Datos}

La información cuantitativa que se utiliza en el estudio de investigación proviene de diversas fuentes oficiales nacionales, periodo 2007 - 2016, derivando así datos confiables para el respectivo análisis posterior. Dentro de las fuentes a las que se recurrió se tienen: Censo Nacional de Población y Vivienda 2010 realizado por el Instituto Nacional de Estadísticas y Censos (INEC), Banco Central del Ecuador (BCE), Servicio de Rentas Internas (SRI) y Registro Interconectado de Programas Sociales (RIPS) de la Secretaría Nacional de Planificación y Desarrollo (SENPLADES). Se tomó información acerca de las siguientes variables (ver Tabla 1): 


\section{Tabla 1}

Variables de análisis

\begin{tabular}{|c|c|c|}
\hline Variable & Enfoque & Definición \\
\hline Sectorización de la población & Social & $\begin{array}{l}\text { Construido a partir de la evolución } \\
\text { demográfica y la participación rural y } \\
\text { urbana. }\end{array}$ \\
\hline Nivel de alfabetismo & Social & $\begin{array}{l}\text { La información se recolectó del Instituto } \\
\text { Nacional de Estadísticas y Censos. }\end{array}$ \\
\hline $\begin{array}{l}\text { Tasa de Afiliados Seguridad } \\
\text { Social }\end{array}$ & Social & $\begin{array}{l}\text { La información se recolectó del Instituto } \\
\text { Nacional de Estadísticas y Censos. }\end{array}$ \\
\hline PIB del Cantón & Económica & $\begin{array}{l}\text { La información fue recolectada en el Banco } \\
\text { Central del Ecuador. }\end{array}$ \\
\hline Nivel de ingresos por Sectores & Económica & $\begin{array}{l}\mathrm{La} \text { información fue recolectada en el Banco } \\
\text { Central del Ecuador. }\end{array}$ \\
\hline $\begin{array}{l}\text { Volumen de Crédito de } \\
\text { Desarrollo Humano }(\mathrm{CDH})\end{array}$ & Económica & $\begin{array}{l}\text { Construido a partir de la información } \\
\text { provista por el SENPLADES. }\end{array}$ \\
\hline $\begin{array}{l}\text { Volumen de Bono de Desarrollo } \\
\text { Humano (BDH) }\end{array}$ & Económica & $\begin{array}{l}\text { Construido a partir de la información } \\
\text { provista por el SENPLADES. }\end{array}$ \\
\hline
\end{tabular}

Fuente: Elaboración propia, 2019, basada en INEC (2010), SRI (2018), BCE (2018) Y

SENPLADES (2018).

\subsection{Modelización de la sensibilidad del Producto Interno cantonal respecto a sus sectores económicos}

La econometría aparece como una conjunción de la teoría económica, economía matemática, estadística económica y estadística matemática, con la finalidad de darle un enfoque empirista a los fenómenos económicos (Gujarati y Porter, 2010). El modelamiento econométrico que se ejecuta en la presente investigación toma en consideración el Producto Interno Bruto cantonal y el valor agregado de los sectores económicos con mayor relevancia.

En el estudio se hace uso de los modelos econométricos de tipo datos de panel, debido al tipo de base de información que se ha adquirido. En la ecuación (1) se muestra la nomenclatura de un modelo de datos de panel, la cual está dado por la combinación de variables de corte transversal (coss sectional) tales como: Ciudades, países, capturados en el tiempo (time series). En general, las variables de corte transversal presentan limitantes como: No recogen la relación intertemporal de las series de tiempo y no resuelven problemas de persistencia en el comportamiento de un evento; por otro lado, las series de tiempos no recogen las relaciones causales, por lo que podrían experimentar problemas de multicolinealidad.

$$
y_{i t}=\alpha+\beta x_{i t}+u_{i t}
$$

En los datos de tipo panel es frecuente la presencia de heterogeneidad o diferencias entre las unidades de evaluación, por lo que los modelos de datos de panel se tornan indispensable, puesto que estos contienen importantes herramientas para dicha característica de heterogeneidad (Gujarati y Porter, 2010). Entre los tipos de modelos de datos de panel más utilizados, se encuentran: Pool de datos (ver ecuación 1), efectos fijos, efectos aleatorios (Wooldridge, 2010).

$$
\begin{aligned}
y_{i t} & =\alpha+\beta x_{i t}+\mu_{i t}+v_{i t} \\
\mu_{i} & =\sum_{i=1}^{T-1} \mu_{i} Z_{i} Y \varphi_{i}=\sum_{i=1}^{T-1} \varphi_{i} K_{i} \\
y_{i t} & =\alpha+X_{i t} \beta+u_{i t} \\
U_{i t} & =\mu_{i}+v_{i t}
\end{aligned}
$$


Las ecuaciones 2 y 3 reflejan la nomenclatura de los Modelos Efectos Fijos (MEF) y Efectos Aleatorios, respectivamente. El MEF parte del supuesto que el coeficiente de la constante varía a través de los agentes o del tiempo, además permite analizar las variaciones intertemporal $\mathrm{y} / \mathrm{o}$ transversales por medio del término de intercepción, como los modelos ANCOVA. Se asume que si CORR (ui, X) es distinto de cero, el MEF sería el modelo adecuado. Por otro lado, los Modelos de Efectos Aleatorios es también conocidos como Modelo de Componentes de Error, y considera a "ui" como variable aleatoria, en contraste con el MEF este modelo no crea variables dicotómicas $\left(\mu_{i} y \varphi_{i}\right)$, sin embargo, los componentes "ui" se dejan que varíen de manera aleatoria tanto para los agentes como para el tiempo.

Para el estudio de investigación se estima un modelo de datos de panel que mejor se ajuste a los datos y variables a las que se hace referencia. Para dicho modelo se considera como agentes a los cantones, objetos de estudios, y a las variables: PIB cantonal y los ingresos en el sector agrícola, comercial y manufacturero, aquellos que son las principales actividades económicas que ejercen los cantones de estudio. Cabe destacar, que se analiza la sensibilidad que tiene cada uno de estos sectores en el ingreso total medido por el PIB cantonal. La estimación del modelo se especifica a continuación en la ecuación 4:

$$
\log P I B_{\text {cant }}=\alpha+\beta_{1} \log \text { Agri }+\beta_{2} \log \text { Comer }+\beta_{3} \text { Manufactura }+u_{i t}
$$

Además, la utilización de los logaritmos permiten a la variables mencionadas a estacionarizar su comportamiento a través del tiempo, lo cual es importante para robustecer el modelo, también aporta a corregir cualquier problema de linealización entre las variables, y por último, como se trata de logaritmos, se evalúa la sensibilidad entre las variables explicativas y la variable explicada, lo que permite identificar cuán diversificada y/o dependiente se encuentra la economía del Distrito a sus principales sectores económicos, en efecto coadyuva a la toma de decisiones según sea el resultado que arroje el modelo. Por otro lado, las variables sociales son descrita y analizadas en los siguientes apartados.

\section{Análisis situacional del desarrollo económico local en el Distrito 9 del Guayas}

El sector productivo que capta la mayor atención, debido a su importancia en las economías de dichos cantones, es el sector agrícola (INEC, 2016). La producción agrícola es bastante homogénea en los tres cantones, tanto en sus modos de producción como en sus productos, por lo que se vuelve una real competencia entre los actores productivos de aquel sector. Además del sector agrícola, el sector comercial y manufacturero han ganado espacio en sus mercados, por lo que destaca una incipiente diversificación de sus matrices productivas.

Por otro lado, la sectorización por área de la población en los cantones de estudio guarda una gran similitud en sus estructuras, pero con ciertas diferencias en las evoluciones de estas, lo cual incide directamente en la focalización que deben tener las políticas públicas que se vayan a ejecutar en dichas localidades, tales como: Políticas de salud, educación, protección social, entre otras. A continuación, se muestra en la Tabla 2 la evolución de la población que han tenido los cantones clasificado por áreas (urbana y rural): 


\section{Tabla 2}

\section{Evolución demográfica por área}

\begin{tabular}{cccccccccc}
\hline \multirow{2}{*}{ Area } & \multicolumn{3}{c}{ Nobol } & \multicolumn{4}{c}{ Santa Lucía } & \multicolumn{3}{c}{ Daule } \\
& $\mathbf{1 9 9 0}$ & $\mathbf{2 0 0 1}$ & $\mathbf{2 0 1 0}$ & $\mathbf{1 9 9 0}$ & $\mathbf{2 0 0 1}$ & $\mathbf{2 0 1 0}$ & $\mathbf{1 9 9 0}$ & $\mathbf{2 0 0 1}$ & $\mathbf{2 0 1 0}$ \\
\hline \multirow{2}{*}{ Urbano } & 4371 & 6357 & 8256 & 5254 & 6958 & 8810 & 24000 & 31763 & 65145 \\
& $42 \%$ & $43 \%$ & $42 \%$ & $19 \%$ & $21 \%$ & $23 \%$ & $41 \%$ & $37 \%$ & $54 \%$ \\
\multirow{2}{*}{ Rural } & 6153 & 8396 & 11344 & 22088 & 26910 & 30113 & 35000 & 53385 & 55181 \\
& $58 \%$ & $57 \%$ & $58 \%$ & $81 \%$ & $79 \%$ & $77 \%$ & $59 \%$ & $63 \%$ & $46 \%$ \\
\multirow{2}{*}{ Total } & $\mathbf{1 0 5 2 4}$ & $\mathbf{1 4 7 5 3}$ & $\mathbf{1 9 6 0 0}$ & $\mathbf{2 7 3 4 2}$ & $\mathbf{3 3 8 6 8}$ & $\mathbf{3 8 9 2 3}$ & $\mathbf{5 9 0 0 0}$ & $\mathbf{8 5 1 4 8}$ & $\mathbf{1 2 0 3 2 6}$ \\
\hline
\end{tabular}

Fuente: Elaboración propia, 2019, basada en información del INEC (2010).

Uno de los desafíos que enmarca a los tres cantones del Distrito 9 es la educación, en otras palabras, el capital humano. Por tanto se considera este último como protagonista en el desarrollo, específicamente, en la búsqueda de construir los consensos necesarios para la transformación territorial (Delgado, 2018). A nivel mundial, la potenciación del capital humano es el principal requisito para obtener un progreso económico sostenible (Mishan,
1983), debido a eso se han puesto todas las fuerzas hacia el desarrollo sostenido del capital humano. En este caso, el capital humano cualificado en el distrito es escaso, debido a sus altos niveles de analfabetismo en sus territorios. A continuación, en la Tabla 3 se muestra la evolución de las tasas de analfabetismo en los cantones y la comparación con el promedio de la Provincia del Guayas.

Tabla 3

Evolución de la tasa de analfabetismo

\begin{tabular}{ccccc}
\hline Area & Nobol & Santa Lucía & Daule & Guayas \\
\hline 2001 & $13,50 \%$ & $18,90 \%$ & $13,80 \%$ & $7,10 \%$ \\
2010 & $10,70 \%$ & $15,50 \%$ & $9,80 \%$ & $5,00 \%$ \\
Intercensal & $\nabla \mathbf{2 , 8}$ & $\nabla \mathbf{3 , 4}$ & $\nabla \mathbf{4}$ & $\nabla \mathbf{2 , 1}$ \\
\hline
\end{tabular}

Fuente: Elaboración propia, 2019, basada en información del INEC (2010).

Notablemente, Daule ha sido el cantón que mayor ha reducido los niveles de analfabetismo dentro de la jurisdicción del Distrito 9; no obstante, los niveles que muestran los cantones en estudio están por encima del promedio en la provincia, lo que destaca la presencia de mala focalización en las políticas públicas en la educación.

Otra arista importante dentro del desarrollo adecuado del capital humano está la protección social. Ecuador ha tomado diversas políticas enfocadas a aumentar el número de afiliados al seguro general (IESS, 2016), pero esto es solo una parte de la protección social, puesto que los seguros privados también forman parte de tal criterio, por lo que la facilitación en el acceso a dichos seguros tendría un gran impacto en la protección social. 
Además, el seguro campesino privado, también es otro tipo de protección social, enfocado principalmente, hacia el campesinado del país. Este tipo de seguro es realmente relevante para el Distrito 9, debido a que la mayor parte de la Población
Económicamente Activa (PEA) se encuentra ocupada en el sector agrícola (INEC, 2010), por ende, los campesinos buscan de alguna forma salvaguardar sus vidas con el acceso a este tipo de seguro.

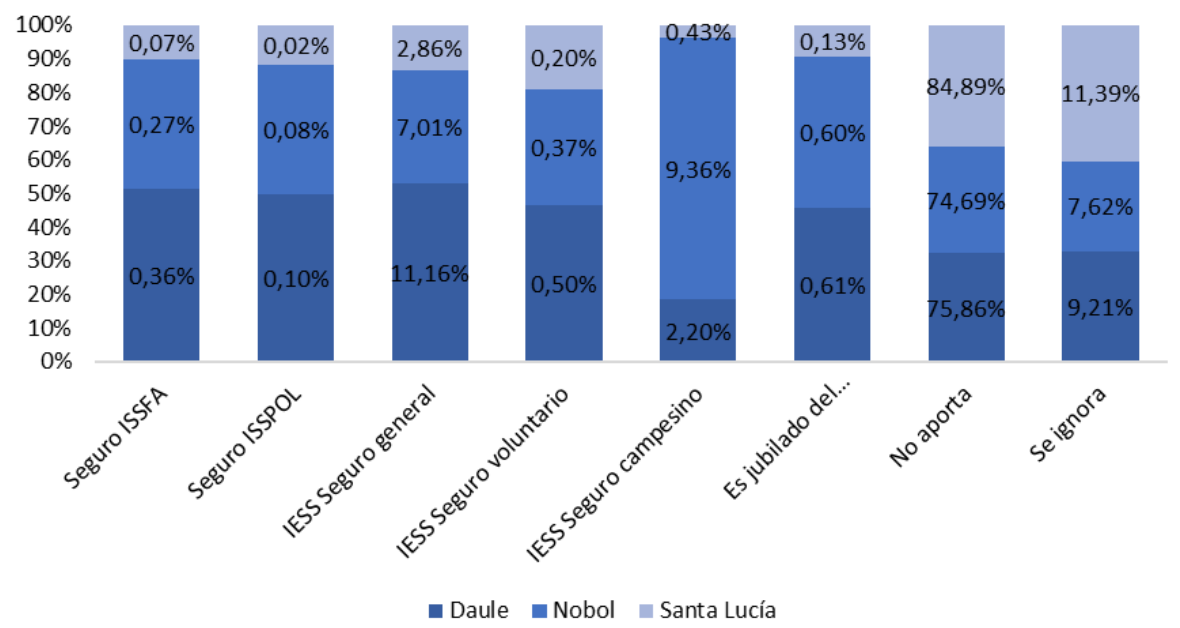

Fuente: Elaboración propia, 2019, basada en información del INEC (2010). Figura II. Población de 12 y más años por aportación o afiliación al seguro

La protección social en el Distrito 9 es muy caótica, tal como se aprecia en la Figura II. Según el último censo poblacional y vivienda reporta que de cada 10 personas de 12 años y más, aproximadamente en promedio 8 persona no realiza ningún tipo de aporte, es decir no registra ninguna aportación a la seguridad social. Contar con algún tipo de seguro es indispensable en el desarrollo de los habitantes de cualquier sociedad, puesto que, al no contar con un seguro, la propensión marginal al consumo tiende a disminuir por la presencia de un gasto tan relevante como es el de la salud.

Por otro lado, una de las políticas públicas que se ha manejado a lo largo de la última década ha sido las transferencias monetarias hacia las personas que más lo necesitan, es el caso del Bono de Desarrollo Humano (BDH) y Pensión Asistencial, así como también el Crédito de Desarrollo Humano $(\mathrm{CDH})$, el cual se observa en la Tabla 4 sus beneficiarios. Estas directrices tienen como finalidad mermar las necesidades básicas insatisfecha por parte de las personas con niveles ineficaces de ingresos. 


\begin{tabular}{cccc}
\multicolumn{5}{c}{ Tabla 4 } \\
Beneficiarios del Crédito de Desarrollo Humano (CDH) \\
\hline Año & Daule & $\begin{array}{l}\text { Beneficiarios } \\
\text { Nobol }\end{array}$ & Santa Lucía \\
\hline 2014 & 3.127 & 264 & 1.386 \\
2015 & 2.452 & 223 & 871 \\
2016 & 2.806 & 208 & 1.221 \\
2017 & 3.240 & 294 & 1.063 \\
$2018^{*}$ & 975 & 104 & 366 \\
\hline
\end{tabular}

*Hasta el mes de junio.

Fuente: Elaboración propia, 2019, basado en los registros del SENPLADES (2018).

La Tabla 4 muestra que la mayor cantidad de beneficiarios en este distrito son aquellos ubicados en el Cantón Daule, seguido por Santa Lucía y, por último, Nobol. La relación que existe entre la cantidad de beneficiarios y la población de los cantones es directamente proporcional. Por otro lado, cabe destacar que el año 2017 ha tenido mucho más beneficiarios que en los años anteriores en los cantones Daule (3.240) y Nobol (294); mientras que, para Santa Lucía fue en el 2014, con 1.386 beneficiarios.

Según los datos que arrojó el último censo de población y vivienda en el año 2010, en Daule de cada 10 personas, 8 son pobres bajo el indicador de pobreza por Necesidades Básicas Insatisfecha (NBI); en Santa Lucía, la situación es mucho más latente, puesto que de cada 10 personas, 9 son pobres bajo el mismo indicador, y, por último, en Nobol, de cada 10 personas, 7 son pobres bajo NBI, lo cual indica que en promedio dentro de la zona del distrito 09 , un $80 \%$ se encuentran en la pobreza por NBI.

A diferencia del Crédito de Desarrollo Humano (CDH), el Bono de Desarrollo Humano y las pensiones asistenciales recaen en mucho más beneficiarios, debido a la naturaleza de este, como se aprecia en la Tabla 5. En el apartado anterior se mencionó los niveles de pobreza que existen en estos cantones, y los niveles de beneficiarios del $\mathrm{BDH}$ ratifican dicha información. En lo que va del 2018, a junio se ha alcanzado casi 14 mil beneficiarios en Daule, 1.7 mil en Nobol y 6.3 mil en Santa Lucía, claramente la diferencia radica en la demografía de cada uno de estos cantones.

Tabla 5

Beneficiarios del Bono de Desarrollo Humano (BDH) y pensión asistencial

\begin{tabular}{cccc}
\hline Año & Daule & $\begin{array}{c}\text { Beneficiarios } \\
\text { Nobol }\end{array}$ & Santa Lucía \\
\hline 2014 & 15.404 & 2.002 & 6.631 \\
2015 & 15.159 & 1.960 & 6.707 \\
2016 & 14.487 & 1.843 & 6.420 \\
2017 & 14.184 & 1.829 & 6.298 \\
$2018^{*}$ & 13.965 & 1.701 & 6.259 \\
\hline
\end{tabular}

*Hasta el mes de junio.

Fuente: Elaboración propia, 2019, basado en los registros del RIPS (2018). 
Una de las soluciones colaterales que se deben implementar con mucha más fuerza es la continua capacitación y asistencia técnica a las personas que perciben el $\mathrm{BDH}$ y/o el $\mathrm{CDH}$, debido a que bajo el acompañamiento especializado, las probabilidades de que los beneficiarios emprendan algún negocio con la finalidad de sostenerse por sí mismo, serán mucho más elevadas, casos ejemplificadores de esta perspectiva está el peruano y salvadoreño. El emprender está bajo el enfoque de desarrollo económico local, así como también la formalidad de los negocios impulsados en el territorio del Distrito 09, puesto que estos dinamizarán las economías domésticas.

En lo referente a las economías del Distrito 09, a nivel cantonal se registra de forma anual el Producto Interno Bruto (PIB) medido a través del Valor Actual Bruto (VAB), una de las formas de cuantificar la economía de dichas ciudades. De forma oficial, el Banco Central del Ecuador es el ente encargado de calcular dichos valores, pero este lo presenta con un rezago de aproximadamente dos años, es decir que la última cifra que se encuentra disponible respecto al PIB cantonal es del año 2016.

En el Cantón Daule, dentro del período 2007 - 2016 la economía ha sufrido varias fluctuaciones, dadas por el comportamiento de sus sectores productivos. Durante los primeros tres años de dicho período, su principal sector fue el comercial, dejando así a un lado el sector agrícola, que es el que absorbe mayor mano de obra en el cantón. Pero, el comercio, tiene una marcada tendencia negativa desde el 2008, mientras que, el sector agrícola, aunque por debajo del comercio, ha tenido un comportamiento más constante, es decir, no ha existido ni un incremento ni un descenso significativo. Bajo el enfoque DEL, la economía de Daule muestra en la Figura III, que el comportamiento de sus sectores está ralentizado, por lo que no se observa a mediano plazo un desarrollo sostenible.

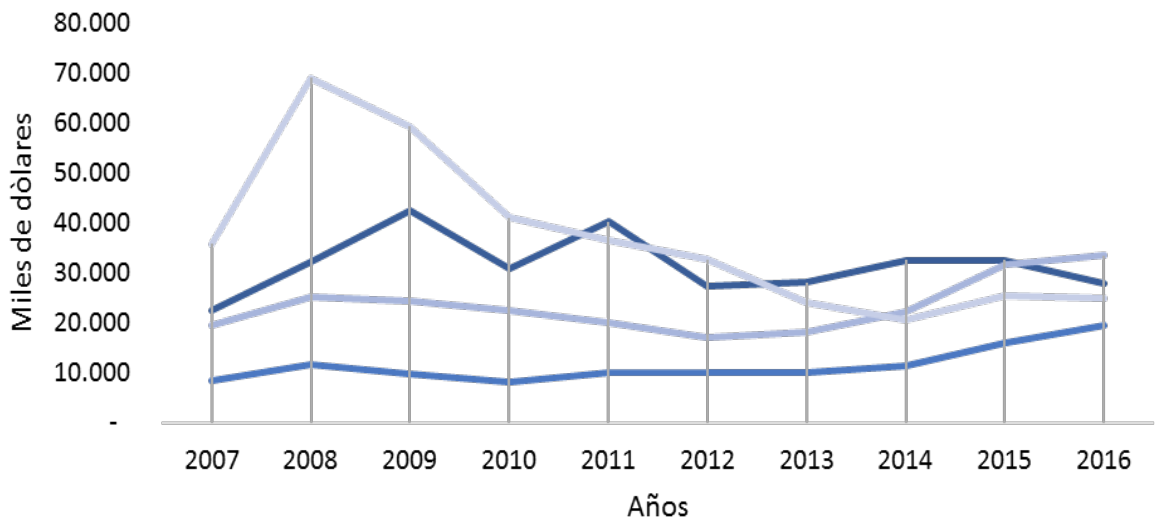

Agricultura, ganadería, silvicultura y pesca $\longrightarrow$ Manufactura $=$ Construcción $\rightleftharpoons$ Comercio

Fuente: Elaboración propia, 2019, a partir del Banco Central del Ecuador (2018). Figura III. Evaluación de los principales sectores de la economía de Daule, periodo $2007-2016$ 
Santa Lucía, con una menor superficie respecto a Daule, cuenta con una economía, netamente, conducida por el sector agrícola, por lo que un decaimiento en dicha arista económica hará que la economía cantonal entre en un estado de crisis. En otras palabras, si el desarrollo solo se basara en los niveles de ingresos que percibe una sociedad, este cantón tendría un camino, en lo económico, totalmente sostenible, puesto que el sector que conduce la economía tiene implícito diversos riesgos no controlables, tal como: Fenómenos naturales.

En contraste a Daule, Santa Lucía mantiene como sector importante a la industria manufacturera, pero frente al agrícola no guarda significancia, como se puede observar en la Figura IV, por lo que se vuelve apriorístico la toma de políticas públicas que dinamicen la economía de Santa Lucía.

25.000

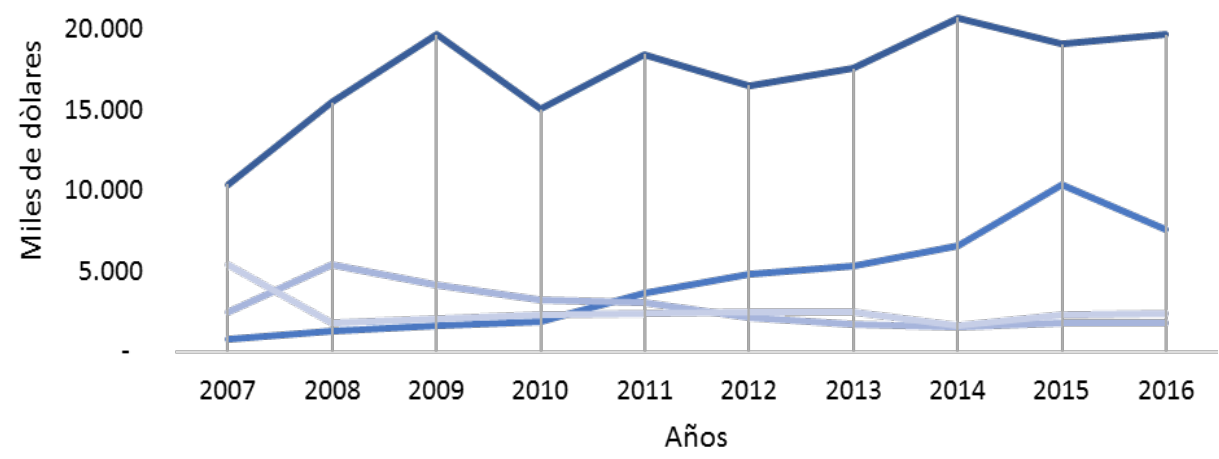

_Agricultura, ganadería, silvicultura y pesca Manufactura Construcción Comercio

Fuente: Elaboración propia, 2019, a partir del Banco Central del Ecuador (2018). Figura IV. Evaluación de los principales sectores de la economía de Santa Lucía, periodo $2007-2016$

Finalmente, el Cantón Nobol, muy homogéneo a Santa Lucía en lo que a su economía se refiere, pero esta cuenta con una particularidad en el comportamiento del último año del periodo en estudio, debido a que el sector manufacturero desarrolla un comportamiento peculiar en relación con los demás años, tal como se muestra en la Figura V. Respecto al sector que sostiene la economía de Nobol es la economía agrícola. 


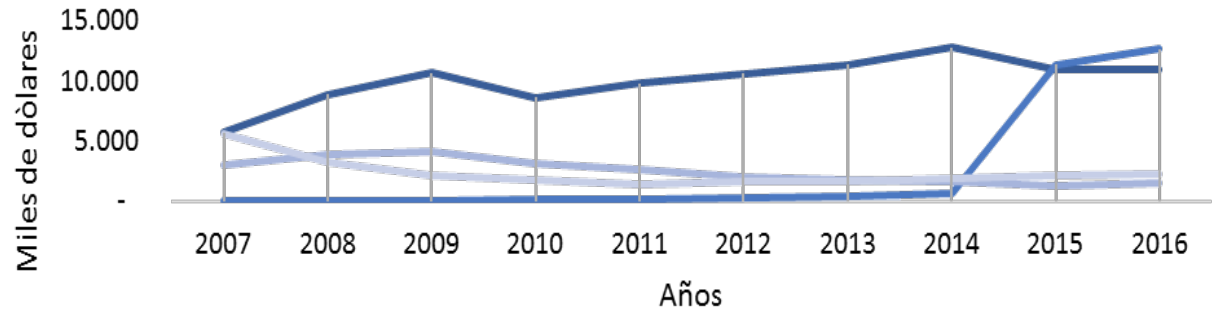

Agricultura, ganadería, silvicultura y pesca

Manufactura

Construcción

Comercio

Fuente: Elaboración propia, 2019, a partir del Banco Central del Ecuador (2018).

Figura $\mathbf{V}$. Evaluación de los principales sectores de la economía de Nobol, periodo $2007-2016$

Aunque, tanto Nobol como Santa Lucía mantienen al sector agrícola como sostén de sus economías, estos perciben niveles bajo de ingresos de ese sector en comparación con los que recibe el Cantón Daule. En promedio, durante el período 2007 - 2016, Daule percibió un $84 \%$ más en los ingresos agrícolas que Santa Lucía, y un 215,6\% que Nobol. Claramente, la economía agrícola de Daule guarda brechas importantes respecto a los niveles de productividad y competitividad, criterios indispensables en el incremento de los márgenes de beneficios.

\section{Resultado de la estimación del modelo}

Para la estimación de la ecuación (4) se hizo uso del lenguaje R, mismo que facilita la manipulación de base de datos $\mathrm{y}$ modelamientos de diversos tipos, en este caso modelos de datos de panel. En el presente estudio de investigación se calcularon los estimadores bajo el modelo de pool de datos (Pooling Model), tal como se muestra a continuación en la Tabla 6.

\section{Tabla 6}

\section{Resultado de las estimaciones del modelo}

\begin{tabular}{lcr}
\hline \multicolumn{1}{c}{ Variables Independientes } & \multicolumn{2}{c}{ Variable dependiente: Log (PIB) } \\
& Coeficientes & \multicolumn{1}{c}{$\boldsymbol{p}$-value } \\
\hline Intercepto & 2,4886 & $(0,08916)$. \\
Log (Agrícola) & 0,3547 & $(0,0640)^{*}$ \\
Log (Comercio) & 0,4394 & $(0,0000)^{* * *}$ \\
Log (Manufactura) & 0,1911 & $(0,0000) * * *$ \\
R-Squared & 0,8951 & \\
N (observaciones) & 30 & \\
\hline
\end{tabular}

Nota. $* * *$ significancia al $1 \%, * *$ significancia al $5 \% \mathrm{y} *$ significancia al $10 \%$.

Fuente: Elaboración propia, 2019. 
El modelo descrito en la Tabla 6 contiene las valoraciones de los coeficientes formulados en la ecuación (4), estos han sido calculados a través del mejor modelo estático de datos panel, específicamente, bajo un pool de datos, puesto que fue el que mejor estimó a los coeficientes, arrojándolos todos de forma significativo al modelo. En otras palabras, los coeficientes o estimadores del modelo son estadísticamente significativo y explican el comportamiento del crecimiento del Producto Interno Bruto del Distrito 9.

En este sentido, haciendo referencia a las economías en su conjunto (Distrito 09), se observa que el crecimiento del Producto Interno Bruto cantonal es sensible a los cambios que puedan sufrir los sectores objeto de estudio. En efecto, entre las tres áreas productivas descritas como principales para dichas economías, a la que mayor sensibilidad tiene el PIB es al comercio, aun siendo el sector agrícola el que absorbe la mayor cantidad de mano de obra. En otras palabras, el PIB en el Distrito 09 guarda una mayor dependencia hacia el comercio, por lo que cualquier política pública y/o económica que incida en el comercio interno, este repercutirá en la economía del Distrito.

Asimismo, respecto al sector agrícola, también existe un grado de sensibilidad del PIB del Distrito, tomando en cuenta los efectos inobservables entre cantones, hacia este sector, así como hacia el manufacturero.

\section{Conclusiones}

El enfoque teórico del Desarrollo Económico Local ha sido llevado a la práctica mediante algunos instrumentos, pero el principal ha sido la subdivisión de la administración pública, o descentralización del Estado. Mediante el Código Orgánico de Organización Territorial, Autonomía y Descentralización (COOTAD), Ecuador creó nuevas facultades que tienen los Gobiernos Autónomos Descentralizados Municipales y Parroquiales, las cuales están enfocadas hacia el cumplimiento de los objetivos propuestos como Plan Nacional de Desarrollo (anteriormente Plan Nacional del Buen Vivir), y estos guardan estrecha relación con los Objetivos de Desarrollo Sostenible (ODS) propuesto por la CEPAL, pero con un plazo mucho mayor de cumplimiento. Esto verifica la pertinencia que existen entre las naciones y los organismos internacionales por la búsqueda del desarrollo.

En el caso ecuatoriano aún no existe la sinergia necesaria entre las políticas públicas y las diversas ventajas comparativas que posee el país gracias a la ubicación geográfica en la que se encuentra. Estas políticas han estado mal focalizadas, lo cual ha creado brechas económicas a nivel distrital, pero en las cuales sus integrantes poseen características similares. Un caso particular, es el Distrito 9 de la Provincia del Guayas, objeto de estudio de la investigación, en el cual se observan amplias brechas en el comportamiento económico en sus sectores, sobre todo en el sector agrícola, el cual es de vital importancia, puesto que es el que absorbe mayor mano de obra en el Distrito.

El Desarrollo Económico Local (DEL) desde una perspectiva integral, cualifica algunos criterios necesarios para el mejoramiento del bienestar de la sociedad. El Distrito 9, no posee una protección social (medida por los habitantes que cuentan con algún tipo de seguro) en la que los trabajadores e individuos en general puedan desarrollar sus actividades normalmente, esto, además, disminuye la propensión marginal al consumo, debido a que aparecería un nuevo rubro (costoso en algunos casos) como es la salud privada, sobre todo por la actividad que realizan la mayoría de los habitantes. De manera análoga, sucede con la educación en estos cantones, puesto que sus niveles de alfabetismos son realmente precarios respecto al promedio de la provincia.

En suma, el Desarrollo Económico Local en el Distrito 9, tomando en consideración los indicadores sociales y económicos plasmados en esta investigación, se encuentra en una situación que amerita la implementación de políticas públicas y económicas inmediatas, 
con una focalización eficiente que busque la potenciación de las actitudes y aptitudes de los individuos de aquella sociedad. El desarrollo de estas es el requisito indispensable para emprender el camino hacia un desarrollo integral y sostenible, en otras palabras, el mejoramiento del capital humano es la que define la sostenibilidad en el desarrollo de las sociedades.

\section{Referencias Bibliográficas}

Aghón, G., Alburquerque, F., y Cortés, P. (Comps.) (2001). Desarrollo económico local y descentralización en América Latina: Análisis comparativo. Santiago de Chile: CEPAL/GTZ. América Latina. Recuperado de https://repositorio.cepal.org/

Alburquerque, F. (2004). Desarrollo económico local y descentralización en América Latina. Revista de la CEPAL, (82), 157-174.

Banco Central del Ecuador - BCE (2018). Boletines Macroeconómicos. Banco Central del Ecuador web site. Recuperado de https://www.bce.fin.ec/.

Cárdenas, N. (2002). El desarrollo local su conceptualización y procesos. Provincia, (8), 53-76.

Chang, H-J. (2004). Retirar la escalera. La estrategia del desarrollo en perspectiva histórica. Madrid, España: Instituto Universitario de Desarrollo y Cooperación.

Chang, H-J. (2010). 23 cosas que no te cuentan sobre el capitalismo. Madrid, España: Debate.

Delgado, J. C. (2018). Dimensión prospectiva del actor local en el talento territorial. Revista de ciencias Sociales (RCS), XXIV(2), 83-93.

Durán, J. (2015). Una aproximación a la rela- ción entre el desarrollo regional y el emprendimiento en Colombia. Sotavento $M B A$, (25), 92-98.

Gobierno Autónomo Descentralizado de la Ilustre Municipalidad del Cantón Daule. (2015). Plan de Desarrollo y Ordenamiento Territorial del Cantón Daule 2011-2016. Daule, Ecuador: Consejo de Planificación del GAD I.M. del Cantón Daule. Recuperado de https:// www.daule.gob.ec/Portals/0/Transparencia/PlanEstrategico/PDOT DAULE_FINAL_WEB.pdf

Gobierno Autonómo Descentralizado Municipal del Cantón Nobol (2015). Actualización del Plan de Desarrollo y Ordenamiento Territorial del Cantón Nobol. Nobol, Ecuador: Consejo de Planificación del GADM. del Cantón Nobol. Recuperado de http://app.sni. gob.ec/sni-link/sni/PORTAL SNI/ data sigad plus/sigadplusdocumentofinal/0960006180001 PDYOT\%20 NOBOL 15-03-2015 17-44-51.pdf

Gobierno Autónomo Descentralizado $\mathrm{Mu}$ nicipal del Cantón Santa Lucía (2015). Plan de Desarrollo y Ordenamiento Territorial del Cantón Santa Lucía 2014-2025. Santa Lucía, Ecuador: Consejo de Planificación del GAD I.M. del Cantón Santa Lucía. Recuperado de http://app.sni. gob.ec/sni-link/sni/PORTAL_SNI/ data sigad plus/sigadplusdocumentofinal $/ 0960002000001$ PDyOT $\% 20$ SANTA \%20LUC\%C3\%8DA\%20 20142025 A C T UA L I Z A DO 2016 16-04-2016 11-52-35.pdf

Gujarati, D., y Porter, D. (2010). Econometría. México: McGraw-Hill/Interamericana Editores, S.A.

Instituo Ecuatoriano de Seguridad Social IESS (2016). Boletín Estadístico de la Seguridad Social. Quito, Ecuador: Publicaciones IESS. Recuperado de https://www.iess.gob.ec/ 
Desarrollo económico local en Ecuador: Relación entre producto interno bruto y sectores económicos

Proaño Chaca, Sonnia Alexandra; Quiñonez Alvarado, Erika Sonia; Molina Villacís, Carolina Jennifer y Mejía Flores, Omar Gabriel

documents/10162/8421754/BOLETIN+ESTADISTICO+22+2016?ver$\underline{\text { sion }=1.0}$

Instituto Nacional de Estadísticas y Censos - INEC (2010). Censo de Población y Vivienda. Quito, Ecuador: Publicaciones INEC. Recuperado de https:// www.ecuadorencifras.gob.ec/base-de-datos-censo-de-poblacion-y-vivienda-2010/

Instituto Nacional de Estadísticas y Censos INEC (2016). Encuesta de Superficie y Producción Agrícola Continua [ES$P A C]$. Quito, Ecuador: Publicaciones INEC. Recuperado de https://www. ecuadorencifras.gob.ec/documentos/ web-inec/Estadisticas agropecuarias/ espac/espac-2016/Informe $\% 20$ ejecutivo $\% 20$ ESPAC 2016.pdf

Maldonado, A. (2000). Descentralización y desarrollo económico local una visión general del caso de Colombia. Chile: Proyecto CEPAL/GTZ, CEPAL.

Mishan, E. (1983). Growth: The price we pay. Barcelona, España: Ediciones Orbis, S.A.

Organización Internacional del Trabajo (OIT)
(2018). Desarrollo Económico Local (DEL). Recuperado de https://www. ilo.org/empent/areas/WCMS 141191/ lang--es/index.htm

Quispe, G., Ayaviri, D., y Maldonado, R. (2018). Participación de los actores en el desarrollo local en entornos rurales. Revista de Ciencias Sociales (RCS), XXIV(3), 62-82.

Secretaría Nacional de Planificación y Desarrollo - SENPLADES (2018). Registro Interconectado de Programas Sociales [RIPS]. Recuperado de http://www. rips.gob.ec/Rips/home.jsf

Servicio de Rentas Interna - SRI (2018). Saiku Next Generation. Recuperado de https://declaraciones.sri.gob.ec/saiku-ui/

Vázquez, A. (2000). Desarrollo económico local y descentralización: Aproximación a un marco conceptual. Santiago, Chile: Proyecto CEPAL/GTZ, CEPAL

Wooldridge, J. (2010). Introducción a la econometría. Un enfoque moderno. México D.F.: Cengage Learning Editores, S.A. 